\title{
VOYELLES, CONSONNES ET STROPHES \\ Une lecture de LXTROHP de Camille Goemans
}

à L

«Certains mots prennent figure d'une belle femme sans corps.»

Camille GOEMANS, Réflexions.

Figure discrète du surréalisme belge, Camille Goemans n'aura guère consenti qu'au rôle effacé de médiateur ${ }^{1}$, quitte à faire oublier l'importance et l'originalité de son apport personnel. Il est vrai que la propagation à Paris des surréalistes belges - et en Belgique du surréalisme général - lui doit beaucoup, que ce soit par les galeries d'art qu'il a dirigées - en Belgique comme à Paris ${ }^{2}$-, ou par les conférences qu'après même son retrait du mouvement, il a consacrées aux activités surréalistes, conférences dans lesquelles il ne se résignait à aborder sa propre contribution que pour aussitôt l'articuler à la démarche collective du groupe bruxellois. Une telle discrétion répond autant, chez Goemans, à une éthique du renoncement, sans doute liée à sa complexion personnelle, qu'à une option "esthétique» délibérée, directement reliée à l'un des enjeux essentiels du surréalisme (suivi en toute rigueur par peu de ses adeptes), appelant à la dissolution de l'individuel dans le collectif, et de la poésie livresque ou de l'art d'exposition dans l'expérience passionnelle de la vie même

1 Voir sur ce point le début de sa conférence sur l' «Expérience du surréalisme» (Euvre. Bruxelles, De Rache, 1970, p.199).

2 Pour rappel : Goemans ouvre en 1926, avec Geert van Bruane, une galerie à l'enseigne de «La Vierge Poupine» (qui organisa notamment une exposition retentissante des œuvres du peintre naïf Edmond de Crom) avant d'ouvrir à Paris, en 1927, la célèbre Galerie Goemans, où seront entre autres exposés Arp, Dali, Yves Tanguy, Magritte. Après quoi, il co-dirige en 1942, à Bruxelles, la galerie Lou Cosyn, puis anime à Anvers, à partir de 1946, la Galerie Artes (couplée avec la revue du même nom). 
(«Plutôt la vie, dit la voix d'en face.» ${ }^{3}$ ) Au poète, à l'artiste, il revient de s'effacer afin que l'œuvre, libérée de ses attaches personnelles, vive de sa vie propre et puisse exercer ses effets en dehors de la sphère culturelle. Camille Goemans appartient ainsi à cette frange réduite des surréalistes qui ont fait du surréalisme un principe d'existence, et non l'alibi d'une renommée littéraire ${ }^{4} \mathrm{De}$ cette «volonté exemplaire de dissimulation» 5 témoigne parmi d'autres signes - comme le «retranchement» complice dans lequel il s'est situé après $1930^{6}$ — sa résistance à entrer dans les circuits de l'édition. Peu enclin à sacrifier au rituel de la littérature ses exigences d'écriture, Goemans aura préféré emprunter des voies de traverse, dussent-elles même conduire à l'impasse ou à l'oubli. La plupart de ses textes furent diffusés sur des supports précaires - comme le tract, la revue à feuilles volantes ${ }^{7}$ ou la conférence - quand ils n'étaient pas simplement véhiculés sur manuscrit à l'intérieur du cercle électif de quelques proches. C'est dire, malgré le titre de l'édition De Rache ${ }^{8}$, que sa production compose à peine une Euvre, si l'on entend par là un ensemble organique de textes voués par avance au rassemblement et à la publication. Il n'empêche : aussi dissimulé s'est-il voulu, Goemans reste à coup sûr, avec Nougé, l'un des surréalistes belges les plus lucides (il faut relire, pour leur esprit de rigueur subversive, ses conférences et ses textes théoriques), mais aussi celui qui s'est livré aux expérimentations les plus intenses, la plupart menées au point d'articulation de l'image et du texte. On songe entre autres à l'entreprise du «Sens Propre», conduite en collabo-

${ }^{3}$ Paul Nougé, Réponse d̀ une enquête sur le modernisme (1924). En écho évident à «Plutôt la vie», poème d'André Breton - «voix d'en face » recueilli dans Clair de Terre (Paris, Gallimard, 1978, coll. Poésie, p.73).

${ }^{4}$ Il n'est pas inutile de relever que cette frange réduite contient la plupart des surréalistes belges, moins enclins que leurs confrères parisiens à préserver leur aura d'auteurs. Se réclamant des surréalistes sans œuvre (tels Vaché ou Rigaut), Breton, Eluard ou Aragon ne cessent pas d'annoncer leur retrait de la scène littéraire, avant d'y reparaître avec un recueil nouveau.

5 Article «Camille Goemans» de l'Alphabet des Lettres belges de langue française. Bruxelles, Association pour la Promotion des Lettres belges de la Langue française, 1982, p.241.

6 «Je me suis retranché du groupe surréaliste dans les environs de 1930. Je n'ai plus été mêlé directement aux activités surréalistes proprement dites. Cependant, je suis resté individuellement en relation avec de nombreux surréalistes, dont la plupart sont encore de mes amis.» («Expérience du surréalisme», dans Euvre, op.cit., p.199).

${ }^{7} \mathrm{Par}$ exemple Correspondance. 
ration étroite avec Magritte ${ }^{9}$, et plus encore peut-être à LXTROHP ${ }^{10}$, qui retiendra, en défi, notre attention dans les pages qui suivent.

«Recueil»11 inédit du vivant de Goemans, LXTROHP remonte à 1927, trois années avant que le poète ne s'écarte du surréalisme. La notice de l'édition De Rache révèle que sa composition s'inscrivait dans un projet de collection, conçu avec Nougé et Magritte, que devait prendre en charge l'éditeur bruxellois Henriquez. Projet avorté, comme tant d'autres, la collection se réduisant finalement à une seule plaquette, de Nougé (alias Clarisse Juranville), Quelques écrits et quelques dessins. Mais ces précisions bibliographiques importent peu au regard de la facture adoptée par le poème : dix pages - ou mieux : dix planches 12 - comportant chacune six blocs de texte couplés deux à deux ou disposés trois à trois, selon qu'on adopte une lecture balayant la page de gauche à droite ou de haut en bas. L'ensemble imprimé en capitales, sous forme de caractères romains demigras, sans ponctuation et souvent sans accents ${ }^{13}$. Dès sa prime appréhension par le lecteur, une telle organisation de la matière textuelle confère à LXTROHP un aspect à la fois compact et éclaté, qui tient d'une part à la graisse des caractères, à l'emploi des capitales et à l'effet-bloc de chaque fragment, et, d'autre part, à la distribution en séquences nettement séparées ou aérées par de larges blancs. Mais elle contribue surtout à autonomiser la forme typographique - exemptée de son devoir de transparence - et à instituer le réseau des lettres en facteur primordial de la signification ou, ce qui revient au même, du fonctionnement poétique. En sorte que, d'entrée de jeu, LXTROHP s'avère plus directement tributaire d'une rhétorique du signifiant typographique, tablant

${ }^{8}$ Edition posthume (Goemans est mort en 1960). Voir la note de l'éditeur : «Il s'imposait [...] de sauvegarder une œuvre qui, dans l'état de dispersion où elle se trouvait, risquait de se perdre» (op.cit., p.3).

9 Je me permets de renvoyer, à propos des tracts du «Sens Propre», à mon étude récemment parue : "«Le Sens Propre». Figures de la défiguration chez Magritte et Camille Goemans". Dans Correspondance, n², décembre 1991.

10 Texte recueilli dans Camille GOEMANS, Euvre, op.cit., pp.25-36.

11 C'est le terme qu'emploie la notice de l'édition De Rache (p.250). Peu à propos, nous semble-t-il, dans la mesure où il s'agit moins là d'un ensemble de textes que d'un seul texte, répondant à la même logique d'écriture.

$12 \mathrm{Ou}$ onze planches, si l'on admet - comme nous y inciterons - que le «titre» fait office de texte préliminaire et germinal.

$13 \mathrm{Si}$ les «E» majuscules sont le cas échéant pourvus d'accent, grave ou aigu, et le vocatif «ô" chapeauté par un accent circonflexe, la préposition 
sur des effets d'espace et d'inscription, que d'une rhétorique du signifié qui s'en tiendrait aux mots et à leur relation réciproque à l'intérieur de la chaîne textuelle. Cette disposition particulière du texte, dont le mode d'expression passe «d'abord» par un mode d'exposition, mérite qu'on s'attarde à la resituer - à grands traits - dans le cadre général des expérimentations littéraires portant sur la substance typographique. Ce n'est qu'une fois dégagées ses conditions de possibilité qu'elle retrouve toute sa force de rupture et d'invention. Comme aussi, on le verra, son pouvoir «d'étrangéifier» le discours poétique et de communiquer à sa forme extérieure la dimension célébrative et sensuelle qu'en l'occurrence il contient ${ }^{14}$.

\section{Retour de la lettre}

A s'en tenir au contexte immédiat de l'expérience tentée par Goemans, force est de reconnaître qu'il emprunte une voie peu suivie par ses pairs, pour autant qu'on veuille bien cerner son orientation propre et les ressources originales qu'elle met en œuvre. Le traitement expressif de la typographie n'est certes pas chose neuve. Des vers rhopaliques de l'Antiquité ou des «carminata figurata » médiévales aux calligrammes d'Apollinaire - en passant, étape décisive, par Un Coup de dés -, les tentatives se sont multipliées pour doter le dispositif typographique de valeurs expressives. Mais dans la plupart des cas recensés, la lettre figurative reste prise, vis-à-vis du texte lui-même, dans un rapport de redoublement idéogrammatique, qui n'autorise son autonomie visuelle que pour la mettre rétroactivement au service du message discursif. Autrement dit, la promotion de la lettre ne s'effectue qu'en surface : dès que le texte prend corps, au fil de sa lecture, il absorbe son enveloppe typographique et la retourne en simple extériorité mimétique. Plus près de Goemans, le collage dadaïste aura sans doute progressé en direction d'une typographie non iconique, mais pour l'investir en revanche d'une fonction méta-génésique et iconoclaste (elle est signe du bricolage qui a présidé à l'élaboration du texte, et forme manifestaire d'une

«A» en est chaque fois privée, sans doute pour occasionner un jeu d'incertitude grammaticale avec l'auxiliaire.

14 Ce partage entre extériorité et intérioritế du texte n'est ici adopté que par commodité préliminaire. On aura l'occasion d'insister, par la suite, sur le rapport indécidable qui se noue dans LXTROHP entre le dedans et le dehors, revenant à saper la pertinence - déjà boiteuse par ailleurs - d'une telle distinction. 
rupture avec l'esthétique du livre normatif ${ }^{15}$ ). Pris entre deux moments d'intense activité littérale - d'un côté la «révolution typographique» décrétée par Marinetti (relayé par Tzara), de l'autre «la dictature lettriste» d'Isidore Isou -, le surréalisme ralentit cette évolution. C'est que le Sens occupe le devant de la scène poétique et que le ductus de l'écriture automatique ne saurait se laisser conduire par des préoccupations d'agencement graphique (le Sens congédie les signes). Sans doute relève-t-on ici et là - chez Breton dans Clair de terre (1923) ${ }^{16}$, chez Aragon dans Le Paysan de Paris (1926), chez Eluard dans Quelques mots qui jusqu'ici m'étaient mystérieusement interdits (1937) ou chez Nougé dans La Publicité transfigurée (1925) ${ }^{17}$-, plusieurs essais de typographie foisonnante, mais ces démarches, d'ailleurs occasionnelles, restent pour la plupart inscrites dans la tradition du collage dadaiiste. Seuls les tracts et les pages de revues donneront libre cours au jeu des caractères (mais ici encore l'intention proclamatoire l'emporte sur la visée esthétique) ${ }^{18}$. Bref, à l'heure du surréalisme, la lettre stagne ou sert, dans la majorité des cas, des enjeux étrangers à sa propre émancipation.

15 Notons au surplus que le geste du collage typographique s'accorde chez les dadaïstes avec une référence aux formes de l'affiche publicitaire et de l'enseigne, qui exhibe elle aussi leur refus du texte légitime, dit «littéraire».

16 Après s'être livré dans Mont de Piété (1919) à des jeux d'espacement proches des premières expérimentations de Pierre Reverdy, Breton n'est revenu qu'à quelques reprises à des essais de poésie typographique (si l'on excepte les collages de coupures de presse, notamment dans Poisson soluble). Les deux plus remarquables, qui entretiennent un rapport spéculaire, figurent dans Clair de Terre, d'abord sur la couverture dont «l'impression [...] utilise pour le nom de l'auteur, le titre et la date, un cliché où des noirs viennent remplir les intervalles séparant les lettres. [...] Ce renversement du rapport de la lettre à son support est ainsi, pour l'œil, mimétique de celui inscrit dans le titre» (M. Bonnet, «Le regard et l'écriture». Dans André Breton. La Beauté convulsive. Catalogue d'exposition, Centre Georges Pompidou, 1991, pp.33-34). L'autre, à mon sens plus remarquable parce qu'il réduit la fonction mimétique et renvoie surtout au matériau typographique lui-même, consiste dans l'impression verticale, isolé insulairement en pleine page, du seul mot «ILE» (op.cit., p.76), dont la forme à effet de relief en creux n'est pas sans évoquer l'œil des caractères.

${ }^{17}$ Pour une lecture sociocritique de ce recueil, voir dans le présent dossier de Textyles, l'article de M. Biron «Le refus de l'œuvre chez Paul Nougé». J'aurai l'occasion de revenir plus loin sur le caractère exemplaire, encore qu'ambigu, de l'expérience menée par Nougé (voir note 36).

18 Sur la fonction proclamatoire des expériences typographiques menées dans les revues surréalistes, voir, par exemple, J.-P. Bertrand, "Jeux typographiques, enjeux institutionnels dans Littérature». Dans Les Mots la Vie 
Naturellement, du moins dans sa réflexion théorique, Goemans ne rompt pas avec la doctrine générale, comme en témoigneront ses Notes sur la poésie et l'expérience (1934). Non seulement il y reconduit la conception surréaliste d'une subordination de l'écriture aux pulsions de l'écrivain, mais encore il y opère entre l'ordre des mots et l'ordre des signes un partage radical ${ }^{19}$, revenant à faire des premiers les éléments moteurs d'une expérience incontrôlable, et des seconds les objets inertes d'une opération maîtrisée qui «ne pourrait jamais se retourner contre le poète» ${ }^{20}$. Nœud poétique par excellence, le mot est une entité sémantique; par quoi il se prête, non au calcul, mais à l'investissement d'imaginaire. Le signe, en revanche, s'y soustrait : étant l'objet d'une opération formelle, il est «proposé à notre clairvoyance» 21 et, comme tel, il ne peut engendrer ce dérapage de sens, cette échappée du sens qui sont au principe de toute «image». Quand la forme-signe dirige le discours, celui-ci ne saurait s'égarer ni s'affranchir de la vigilance de l'auteur (à l'abri dès lors de tout risque, mais aussi bien de cette chance, si précieuse à Goemans, d'entrevoir sous sa plume «une sorte d'intuition de soi-même»22).

Ce qui vaut pour l'agencement formel des mots entre eux devrait en principe valoir pour leur forme d'agencement intérieur. A suivre Goemans, la lettre tomberait doublement sous le coup de sa défiance. Signe de signe, pur artefact, elle est en-deçà du mot, hors sens, irréductible opacité. Aussi, promue à l'avant-plan, ne risquerait-elle pas d'empêcher l'ouverture métaphorique des mots, et par suite d'entraver le mouvement même du texte qu'ils animent ? A ce risque, LXTROHP répond : en s'y exposant. D'un côté, le signifiant typographique s'y voit placé en évidence et le texte soumis à une distribution spatiale. Mais surtout, comme on s'y attardera, les mots font l'objet d'un travail de coupe, de césure, d'écartèlement, qui compromet leur intelligibilité, rompt leur cohésion littérale et qui, du même coup, exhibe leur constitution graphématique, celle d'un «corps complexe, composite, contradictoire» 23 . Et cela le plus souvent sans respecter leur

(Publications du groupe Eluard de l'Université de Nice), n5, 1987, pp.87101.

19 «Les mots ne sont pas des signes». (Notes sur la poésie et l'expérience (en coll. avec J. Capuano), dans Euvre, op.cit., p.131.)

20 Op cit., p.130.

21 Loc. cit.

22 Ibid., p.131.

23 Ibid., p.131. 
structure syllabique ou phonique (preuve, entre autres, que le principe de la coupe repose sur la lettre elle-même et non sur le sens ou le son). Soit, par exemple, cette première planche du poème :

LEGERE LEGERE FEUILLE DE CHAIR A L'OMBRE FAIBLE ECUME A L'OMBRE DE CHAIR
LE BEAU COLLIER DE BRAS DE PER LES JE VEUX LA NUIT ME RASSU RER D'EAU DOUCE
DE QUOI TE NOUR RIS-TU MAIN B OUCHE DOUBLE MAIN PARURE DE SEL ET D'ONGLE ESPOIR A TOUTE FIN
JE PENSAIS A ELLE J'ETAIS LE SEUL BOU LANGER QUE DE SABLE DE M UR DE PLUIE FI NE LE SEUL VR AIMENT
NOUS NOUS REN CONTRERONS F RAICHE QUELQ UE JOUR UNE EPINGLE NUE $A$ UX YEUX AU CO EUR J'Y SONGE
A SUPERSTITIEUX SUPERSTITIEUSE FORET LA BOUCLE NOURRIE DE SENS LES SEINS ET CE QUE JE NE PENSE PAS 
On l'aperçoit déjà : l'orchestration graphique du texte chez Goemans n'est pas mise au service d'un quelconque mimétisme. La lettre s'y dégage de toute inféodation au sens ou à la syntaxe, son flottement en bout de ligne ne répond, en tout cas de prime abord, à aucune exigence expressive ni figurative. La rupture qui suspend la formation du mot sous l'œil qui la suit, vaut pour ellemême, dans sa radicalité apparemment gratuite et aléatoire. En somme, Goemans innove à deux titres : d'une part en composant un poème surréaliste résolument hors norme ( $\mathrm{y}$ compris celle qu'il s'est fixée, en théorie, à soi-même), d'autre part en dotant l'agencement typographique d'une fonction sans précédent, qu'il faudra définir, et d'un pouvoir sans réserve sur le déroulement de la chaîne morpho-syntaxique ${ }^{24}$.

Il est possible, par parenthèse, qu'un tel traitement de la substance graphique soit lié chez Goemans à sa proximité avec les arts de l'image. De sa fréquentation assidue de la peinture, en particulier celle de Magritte ${ }^{25}$, Goemans pourrait bien en effet avoir retiré une conscience aiguë de ce que j'appellerais la plastique du texte, c'est-à-dire cette conscience que toute écriture est d'abord acte graphique - tracé, signature : inscription - et tout texte «une exploration d'espace autant, sinon plus, qu'un discours» ${ }^{26}$. De là qu'aussi peu homme de lettres que possible, Goemans soit plutôt un homme de la lettre, attentif à son pouvoir d'articulation et de désarticulation, au "piqué» de son dessin comme à sa capacité à brouiller, par sutures et ruptures, la linéarité plane du sens. Toujours est-il qu'LXTROHP, texte conçu à l'origine pour figurer dans une collection alliant le texte et l'image, se développe sans conteste entre le visible et le lisible, entre l'œuvre-à-voir et l'œuvre-à-lire, ou plutôt à leur point d'inextricable jonction. Il suffit, pour s'en convaincre, de se

24 Pour reprendre les termes et les classements grammatextuels proposés par J.-G. Lapacherie, LXTROHP se situerait donc au «pôle alphabétique abstrait», actualisé aux deux niveaux, "grammatique» et «inscriptique», de la lettre et de la page. Cf. «De la grammatextualité». Dans Poétique, n59, septembre 1984, pp. 283-294.

${ }^{25}$ De tous les peintres surréalistes, le plus irréversiblement porté, à l'inverse ou symétriquement, à concevoir la peinture comme acte poétique à part entière (au sens strict du mot "poétique»), convoquant non seulement les tropes visuels mais aussi les ressources figuratives du langage verbal, par exemple dans ses «Tableaux parlants», dont Goemans soutient du reste que leur appellation vaut pour toute l'œuvre du peintre (voir «Magritte et ses tableaux parlants». Dans CEuvre, op.cit., pp.178-180).

26 A.-M. CHRISTIN, «L'écrit et le visible». Dans L'Espace et la Lettre, Cahiers Jussieu, 3, U.G.E., 1977, p.164. 
prêter au jeu qu'il organise et dont il détient seul, en les montrant sans les exprimer, les règles et les enjeux.

\section{ABCD : l'avant-texte}

S'il est vrai que la règle du jeu semble n'être pas donnée, c'est peut-être parce qu'il manque au lecteur la capacité de la reconnaître là où elle se donne, c'est-à-dire la connaissance du code dans lequel elle est transcrite. Compact, hermétique, ne répondant à aucune logique apparente, le titre du poème fait énigme, et par là s'offre à l'interrogation (sa forme énigmatique appelle au déchiffrement et engage d'entrée de jeu le lecteur dans un travail de décodage voué à traverser l'ensemble du texte) :

\section{LXTROHP NVBQDES YCFIKAG JMUW}

En désordre, c'est l'alphabet - moins une lettre, la «dernière», le $\mathrm{Z}^{27}$. Goemans livre donc, en page de tête d'un texte qui en fera son principal objet de travail prosodique, l'appareil graphématique presque au complet. En vrac, semble-t-il, sans se conformer en tout cas à l'ordre alphabétique et sans l'agencer de manière à ce qu'il forme, même de façon lacunaire ou disparate, une série de mots. L'intitulé défie en outre toute prononciation, en particulier dans sa formule d'attaque (LXT) ; autrement dit, pourvu d'un pareil titre, ce texte est innommable, il ne peut être identifié oralement - preuve administrée, en (c)ouverture, que sa dimension d'objet graphique se veut incontournable. Mais à mieux y regarder, la disposition aléatoire des lettres rencontre d'autres enjeux, plus essentiels. D'abord, son arbitraire fait effet d'alphabet, sans en adopter la forme instituée ${ }^{28}$, et communique au titre la fonction même de l'alphabet, celle de matrice générative, de répertoire des signes d'où s'engendre l'infinité des énoncés (parmi lesquels le poème qui suit). Même s'il la modifie, le titre s'affiche ici d'autant plus comme le code du texte, qu'il adopte la structure informelle du grand code graphique dont tout

27 Expulsé du titre, le $\mathrm{Z}$ fera cependant retour dans le texte (aux pp.28, 30, 31 et 33), mais chaque fois dans des verbes à la deuxième personne du pluriel. ${ }^{28}$ Laquelle est non moins arbitraire. L'arbitraire de l'alphabet de Goemans a peut-être aussi pour enjeu de rendre sensible celui de l'alphabet usuel. 
texte procède ${ }^{29} \mathrm{D}$ 'autre part, le caractère aléatoire de l'alphabet placé par Goemans en tête du poème, contribue à dévoiler sa puissance d'engendrement, en le donnant à voir - à lire comme s'il était déjà, avant même que le texte ne prenne corps, engagé dans le jeu des substitutions, permutations et agencements différentiels dont dérive la chaîne des morphèmes, des mots, des syntagmes, des phrases ${ }^{30}$.

Tout indique en somme que le titre d'LXTROHP surexploite la fonction dont chaque intitulé s'avère en principe investi : celle de nom propre du texte, d'affiche identitaire, bien sûr, mais celle aussi d'embrayeur d'écriture et de lecture (au fronton du poème s'expose l'instance littérale qui commandera son élaboration et sa réception ${ }^{31}$ ). Saturation fonctionnelle portée à son comble lorsqu'on s'avise qu'il remplit cet office non seulement en tant qu'abécédaire déjà emporté dans le mouvement d'épellation textuelle, mais encore en adoptant, de manière asémantique, la forme-bloc qui servira de moule aux séquences dispersées du poème. Et cette forme-bloc n'est rien d'autre, en définitive, que le dispositif minimal de la strophe, dont la

29 Texte avant le texte, l'alphabet «accomplit, disait Barthes, une sorte d'état adamique du langage : c'est le langage avant la faute, parce que c'est le langage avant le discours, avant le syntagme, et cependant, déjà, par la richesse substitutive de la lettre, entièrement ouverte sur les trésors du symbole» («Erté». Dans L'Obvie et l'Obtus. Paris, Seuil, 1982, coll. Tel Quel, p.113.) ${ }^{30}$ Resterait à expliquer l'absence du $\mathrm{Z}$, d'autant plus étonnante que, comme s'y est aventurée Claire Lejeune, «Z est [...] est la signature d'origine authentifiant toute procession génératrice [et] l'étymon génétique, l'architexte auquel l'opération poétique ne cessera plus de se référer» ( $L^{\prime}$ 'Eil de la lettre, Bruxelles, Le Cormier, 1984, p.108). Risquons cependant trois hypothèses. Serait-ce façon d'affirmer, déléatoirement, que tout discours est par avance lacunaire (il tait à proportion de ce qu'il dit) et par nature inaccompli (comme la phrase, le discours est susceptible d'infinie expansion) ? Ou serait-ce plutôt que l'alphabet «adamique» de Goemans expulse avant contagion la lettre maudite, dont la zébrure est signe de transgression (c'est le Z forclos de Sarrasine ou le Z vengeur de Zorro) ? Et s'il revenait au manque d'une lettre de faire trébucher l'alphabet et d'embrayer ainsi le mouvement du texte? Evitons de trancher sur ce point. Le Z, comme toujours, restera lettre close - signe d'énigme.

31 Goemans avait envisagé un autre titre : Consonnes et voyelles, raturé sur le manuscrit. Rature providentielle. Pourquoi ? Parce que les consonnes et les voyelles sont des travestissements phoniques de la lettre, remplaçant le dessin par le son, détournant l'image vers la parole. L'imprononçable du second titre de Goemans garantit sa radicale littéralité, et congédie par avance toute réduction symbolique de la forme typographique du texte. Le visible y révoque le dicible. 
première ligne du titre, en indice, semble balbutier l'anagramme (pour autant que le X laisse entendre l'S qu'il contient) :

\section{XTROHP $=>\quad$ [S]TROPH(E)}

Cette apparition masquée de la [STROPHE] mériterait d'être étudiée de près. Car si elle laisse entrevoir le principe organisateur du texte (qui obéira à l'instance strophique quitte à dérégler la morphologie des mots), elle est également sous-tendue par une réflexion d'ordre générique, touchant à la définition la plus originaire de la forme poétique. En l'inscrivant dans un bloc, en clé du texte tabulaire qui suit, le titre de Goemans semble en effet suggérer que toute strophe, comme a fortiori tout poème, est d'abord un bloc graphique, détaché par un large blanc périphérique : occupation d'espace, découpe noire sur fond pâle avant d'être faisceau de significations et orchestration de rythmes. De la même manière, la déstructuration du mot et de la syntaxe qui préside à l'ensemble du texte contribuera à amplifier visuellement son «effet-poème» (car le propre du vers, c'est le versus : le saut à la ligne, la fracture du flux discursif, l'interruption ou la suspension momentanée du phrasé).

\section{Blocs, boucles et regards}

Unité centrale du poème, la strophe-bloc ordonnera donc la répartition des signes littéraux non seulement aux dépens de l'intégrité des mots - coupés sans égard à leur constitution phonique ${ }^{32}$ - mais aussi jusqu'à suspendre, en certains cas, leurs marques d'accord grammatical. Deux exemples parmi bien d'autres :

STUPEUR ARBR

E DE MES JOUR

S ET CE CRI C

ONTINU DESE

RT SI TON OEIL

ALLAIT ALLAIT S'

TEINDRE JE SE

RAIS AVEUGLE
IL ME SOUVIENT

DE RIRE FLEUR

A MOITIE LE SEI

N ROUGIT LA B

OUCHE Y ETA

IS-TU

32 La coupure (que, par commodite, nous marquerons d'un tiret) ne respecte pas en règle générale les structures syllabiques. Elle peut aussi bien affecter des monosyllabes (D-ES, BLE-U, TU DOR-S, B-IEN) que des diphtongues (GOSI-ER), désarticuler la prononciation des mots (L'A-IR, TROUPE-AU, CLOC-HES) que dégager leur initiale ou leur terminaison (D-EMEURE, $N$ OCTURNE, L'U-NIQUE ; ASTR-E, NOI-R, AMOU-R, VERR-E). Voire déstructurer graphématiquement des concrétions phonologiques (O-UI). 
En d'autres occurrences, la coupure opère même en-deçà du mot, de la syllabe et de la diphtongue, en démembrant ou en désimbriquant des digrammes du type «EЕ» ou «Æ» : «co - eur», «l'o - eil» ${ }^{33}$. Cassant les mots puis les recasant, la typographie rabat ainsi la morphologie et la forme graphique elle-même en géométrie, dont le rectangle vertical des strophes définit le plan et la mesure approximative. Insistons-y en effet: dans LXTROHP, ce n'est pas la rupture qui est obligatoire, mais la strophe-bloc elle-même, laquelle ne déstructure les mots que dans les cas - à vrai dire, les plus nombreux - où le maintien de leur intégrité eût engendré, par excédent de signes, une irrégularisation de la masse graphique. La strophe fonctionne ainsi comme une intaille ou comme l'ail d'une lettre, qui donne forme et format, mais retranche en même temps tout ce qui viendrait à dépasser la limite de sa découpe ${ }^{34}$. La meilleure preuve en est qu'on relève dans LXTROHP plusieurs blocs sans coupe morphologique - quatre au total, dont le premier - , dans lesquels l'espace entre les mots pouvait coïncider avec la taille prosodique. Le quadrillage des strophes opère en somme sur le texte ce qu'en termes de mise en page on appelle une «justification», à savoir la définition de la longueur des lignes d'impression. Passe à la ligne tout ce qui outrepasse la ligne.

Malgré l'hypothèse avancée plus haut, selon laquelle la démarche de Goemans contribuerait, en surdéterminant l'instance strophique, à visualiser la poéticité du texte, on peut penser qu'LXTROHP procède tout autant à un dévoilement en acte de l'arbitraire prosodique, qu'il porte en quelque sorte à sa limite. Jusqu'à l'absurde, au prix de contorsions et de monstruosités morphologiques évoquant les exercices de haute virtuosité verbale requis naguère par les contraintes de la versification, apparemment gratuites mais gratifiantes. La fabrication strophique du

33 Il faut cependant remarquer qu'à l'intérieur des blocs eux-mêmes ces lettres accouplées ne sont pas respectées (Eil s'y écrit «OEIL»). L'explication en est peut-être que de telles formes littérales n'existent pas dans l'épigraphie en romaines, ni du reste dans l'alphabet lui-même.

34 Notons au surplus que ce retranchement ne s'accompagne pas d'un quelconque étirement intrinsèque à la ligne dans les cas où la série des signes ne la couvre pas sur toute sa longueur. En plusieurs occurrences, la dernière ligne du bloc ne comporte même qu'un signe, et dans la majorité des cas elle est nettement moins longue que ses précédentes, lesquelles s'accordent chaque fois à la mesure de la découpe strophique, telle qu'est elle définie par la matrice du titre (dont la dernière ligne est elle aussi près de moitié plus courte que les autres (elle ne comporte que 4 lettres contre 7 pour les trois précédentes). 
poème LXTROHP vaudrait ainsi comme dénonciation du caractère (souvent) «fabriqué» des poèmes en vers réguliers. Ce faisant, Goemans anticiperait, l'intention explicite en moins, l'une des stratégies de «défiguration» mises en œuvre, plus près de nous, par Denis Roche, soumettant lui aussi la convention versifiée à l'épreuve de la ligne brisée et du syntagme éclaté 35 . Ce qui, du même coup, conduirait Goemans à rejoindre en amont, après avoir paru l'en écarter, cette subversion des formalismes dont l'activité surréaliste à Bruxelles avait fait l'un de ses objectifs primordiaux ${ }^{36}$. Mais revenons au corps du texte.

Etagés, étoilés sur la page, les blocs y maintiennent de part en part un même style typographique, à trois déterminations :

35 Cf., par exemple, Eros énergumène ou Dépôts de savoir \& de technique.

${ }^{36}$ Dans le cadre de cette activité, la «défiguration» du texte canonique s'opère également au travers d'une «transfiguration» exercée sur des messages détournés de leur site propre et de leur fonction pragmatique, comme il en va chez Nougé avec les slogans publicitaires. Il est frappant, au reste, de constater que certaines des opérations métagraphiques mises en œuvre par ce dernier dans La Publicité transfigurée (dans L'Expérience continue, L'Age d'homme, 1981) recoupent tendanciellement le dispositif d'LXTROHP. Même si la référence explicite à l'intertexte publicitaire et aux formes de l'affiche ou de l'enseigne - tant dans le titre ou dans l'avertissement dont Nougé a fait précéder les textes que dans les textes eux-mêmes (faits de collage de fragments disposés en corps divers) - oriente encore la rhétorique de la typographie dans le sens d'une démarche à portée subversive et contreinstitutionnelle, il reste qu'à trois reprises au moins (pp.335, 336 et 337) Nougé semble devancer Goemans dans sa tentative de soumettre l'organisation des mots et des phrases au principe du bloc typographique. Deux pages y présentent ainsi chacune un bloc compact de mots en caractères gras et sans espaces intersticiels, blocs disposés dans un cas en ligne continue de minuscules lisibles de gauche à droite (p.335) et, dans l'autre, en lignes verticales de majuscules (p.336). Une troisième expérience (p.337), la plus retorse, alterne deux modes d'agencement littéral (de haut en bas/de bas en haut) et adopte en conséquence la forme d'un boustrophédon vertical et disposé dans le sens inverse d'une lecture normale (les lettres se lisent à rebours et à partir de ce qui apparaît d'abord comme la fin de la séquence). Trois façons, comme dit Nougé (p.335) d'éprouver le regard et de désautomatiser la lecture au profit d'un déchiffrement dont la disposition des caractères détermine les modalités. Au lecteur est ainsi rendue une conscience graphique, qui se subordonne l'intelligibilité sémantique. Il n'est pas impossible que l'exemple de Nougé ait servi de modẻle à Goemans pour la composition d'LXTROHP (rédigé peu après). Mais, quoi qu'il y paraisse, Goemans va plus loin que Nougé sur la même voie : chez lui, les blocs de lettres et les effets-de-fragmentation ne renvoient plus à quelque pratique extérieure, dont l'œuvre poétique détournerait à son propre profit les formes ou les techniques: ils ne renvoient qu'à eux-mêmes, dans leur abrupte apparition sur la page. Par là, ils offrent à la typographie un premier terrain d'expression autonome. 
romain, grande capitale et gras (le tout dans un même corps). Or, ces déterminations n'ont de valeur que relative ou différentielle. Le romain s'oppose en principe à l'italique ${ }^{37}$, comme la rectitude à l'oblique; la capitale au bas de casse et le gras au maigre. Triple jeu d'écarts qui constitue d'ordinaire la typographie en système d'effets rhétoriques, dont la puissance est à proportion de la parcimonie et du discernement que l'auteur-compositeur met à y recourir. Si tout fait écart, l'écart fait norme et s'annule. La continuité stylistique de LXTROHP semble donc sous-exploiter l'appareil typographique et le déchoir de son pouvoir figuratif. A moins qu'elle ne vise d'autres effets, qui seraient moins internes qu'externes à l'élaboration du poème. En d'autres mots, l'écart typographique ne se situe pas dans l'intériorité rhétorique du texte mais intervient plutôt dans son rapport différentiel avec la masse extérieure des autres textes, dont il se détache ou se démarque in absentia. Par là, LXTROHP se rend d'emblée étranger au champ textuel général, sans doute pour affirmer hautement l'irréductibilité de son discours aux formes et contenus routiniers circulant dans ce champ. Affirmation d'autant plus radicale qu'elle adopte en les combinant les deux modes d'hyperbole disponibles en typographie (grandes capitales + gras).

Maximisé, porté au plus péremptoire de sa rupture, l'écart extrinsèque du poème satisfait, dans les limites de sa clôture, à une autre forme d'agencement expressif. Le double emploi des romains et des capitales renvoie plutôt au texte épigraphique, à l'inscription lapidaire, qu'au texte d'encre et de papier ${ }^{38}$. L'écriture de Goemans dans LXTROHP grave, semble-t-il, plus qu'elle ne trace, sculpte plus qu'elle ne dessine : dresse en tout cas sur chaque page six stèles massives, dont le matériau fusionne avec les inscriptions dont il est buriné. Autant de blocs levés, exposant au regard voyageur un texte sans autre ponctuation que la découpe externe de leur bordure. Ceci valant du moins lors d'une appréhension immédiate du poème, avant que ses signes ne s'articulent en mots au fil de la lecture. Car, à peine est-on entré dans le texte - dès le premier bloc - la pesanteur de la stèle s'évanouit en légèreté charnelle, celle d'une «feuille» sur laquelle «l'ombre» et l'«écume» alternent leurs tracés :

37 Encore que ce soit plutôt le contraire : c'est à l'italique qu'il revient de rompre la norme du romain (ce qu'atteste visuellement le fait que l'oblique de la lettre italique se détache parfaitement sur fond romain, alors que l'inverse se vérifie moins).

38 Faut-il rappeler en effet que les inscriptions lapidaires antiques - romaines notamment, d'où le nom du caractère «romain» - n'utilisaient que le romain et la capitale, sans ponctuation? 


\section{LEGERE LEGERE \\ FEUILLE DE CHAIR \\ A L'OMBRE FAIBLE \\ ECUME A L'OMBRE \\ DE CHAIR}

Ouverture méta-textuelle, mais doublement contradictoire, puisqu'elle dément l'organisation «externe» du poème (la «légèreté» deux fois répétée contrastant avec la forme lapidaire, et la «faiblesse» de l'ombre ou de l'écume avec le gras de l'impression) et qu'elle associe, en une coïncidence chromatique qui se maintiendra d'un bout à l'autre du texte, l'ombre et l'écume, le noir et le blanc, la marque et la non-marque (ce qui, sous un autre angle neutralisant la contradiction, pourrait aussi bien figurer dans la structure «interne» du texte, la relation de renforcement réciproque unissant, sur la page, ses blocs très noirs et le large fond blanc sur lequel ils se détachent).

Avant d'entrer dans le jeu rhétorique du poème, restons encore un instant à ce qui n'est pas tant sa périphérie ni son enveloppe formelle que son dispositif d'expression et d'expansion. Selon cet effet paradoxal que connaissent bien, pour l'exploiter constamment, les utilisateurs de traitement de textes, l'agencement par blocs contribue moins à isoler les séquences qu'à multiplier entre elles les possibilités de connexion. Avec leurs six blocs disposés suivant le même ordre, les pages d'LXTROHP définissent un espace ouvert, régi davantage par une topographie réversible et multi-directionnelle que par la successivité d'un enchaînement textuel. Espace à balayer sans doute de gauche à droite et/ou de haut en bas, comme nous l'avons dit, mais aussi en tous sens, l'œil du lecteur-opérateur pouvant à l'envi prélever telle séquence puis la souder à telle autre, sans que leur relation soit prédéterminée par une disposition fixée ou par quelque syntaxe contraignante, qui commanderait à leur succession. Mais ce mouvement de balayage et d'assemblage trouve aussi bien son impulsion dans la construction spécifique des blocs eux-mêmes. S'il est vrai que chacun forme un tout, il s'agit cependant d'un tout fragmentiste, à syntaxe approximative voire agrammaticale 39 et dont les éléments thématiques, malgré leur

39 Deux exemples-limites : «ECOU-TE DERRIERE-TOI L'A BLEUEL'AVENTURIERE-L'OUBLIEE-L'AM-ANT», où l'article devant «bleue» - adjectif substantivé ? - s'élide par contamination avec l'article élidé devant les voyelles $\mathrm{A}$ ou $\mathrm{O}$, au point de faire confusion avec la forme verbale «l'a» et au risque consenti, par conséquent, de l'agrammaticalité. Ou : «QUE 
apparente disparate, sont soumis, à l'échelle du texte entier, à un puissant principe de redondance. Par quoi chaque bloc appelle à sa propre «complémentation», non par adjonction intrinsèque de liens syntaxiques ou de motifs supplémentaires, mais par liaison sémantique et logique avec d'autres, au fil d'une «boucle - nourrie de sens».40 Symétrique du premier, dont on a relevé plus haut le caractère méta-textuel, le dernier bloc du texte sera d'ailleurs tout entier voué à reformuler poétiquement ce mouvement qui va d'un bloc à un autre et opère entre eux des jonctions jamais définitives :

\section{LE VISITEUR E COUTE LES BRUIT \\ S DE LA CHAMB \\ RE AILE COMM \\ E TU MARCHES \\ D'ILE EN ILE}

Dans la «chambre» d'échos du poème, le lecteur n'est-il pas en effet ce «visiteur» à l'écoute, suivant «d'île en île», de bloc en bloc, la marche de cette belle inconnue ${ }^{41}$ dont le texte a tracé le

DIT LA VOI-CI LE REVE», où la coupe strophique tombant sur «voici» dégage sa première syllabe pour en faire un instant le sujet «voi(x)» de «dit». 40 On peut expérimenter ce mouvement de connexion en se reportant à la première page du poème, reproduite plus haut. On y observera par exemple que le motif de la nourriture apparait dans trois des blocs, dont deux se font face au "centre» de la page : «DE QUOI TE NOUR - RIS-TU MAIN BOUCHE [...] PARURE DE SEL» renvoie dans le bloc contigu à « J'ETAIS LE SEUL BOU-LANGER QUE-DE SABLE», suivant un mouvement métonymique (nourrir/boulanger) et métaphorique (sel/sable) réversible, dont le «dernier» bloc reformulera, en l'indexant sur le registre métatextuel du sens et donc du Logos, la logique circulaire : «LA BOUCLENOURRIE DE SENS». Formule qui définit exactement, tout en y étant prise, le processus de liaison, d'aimantation et d'alimentation mutuelle qu'entretiennent les blocs - et qui serait, soit dit en passant, une bonne traduction de ce que les théoriciens de l'information, à la suite de Norbert Wiener, ont appelé le feed back.

${ }^{41}$ Sacha Heydeman, épouse du poète, confie dans un témoignage - passablement confus - sur "[S]a vie avec Camille Goemans», qu'elle a été l'inspiratrice et la dédicataire de «Consonnes et voyelles» (p.22), dont elle situe par erreur la rédaction après le retour de Goemans à Bruxelles, soit après 1930 (dans Espaces Documents XXème siècle, $\mathrm{n}^{\circ} 1$, automne 1973, p.26). Mais cette destination anecdotique du texte importe peu, au regard de son élaboration symbolique (laquelle, on va le voir, dissout l'identité de la destinatrice et brouille les formes et les conventions ordinaires de la communication). 
blason ? Défi à la lecture, LXTROHP est tout autant invitation à lire, comme art de lier.

\section{La lettre errante}

Ne nous abusons pas : une telle organisation, à la fois typographique et topo-graphique, disqualifie par avance toute lecture linéaire et fait, ici plus encore qu'ailleurs, de toute interprétation un acte par avance inaccompli, un processus inachevable. En aucun lieu du texte - pas même à sa fin, aussi marquée soit-elle ${ }^{42}-$, le sens ne se prête à une compréhension globale, qui le convertirait en signification. Il n'en reste pas moins qu'ici et là, «d'île en île», à force d'insistance et de répétition, se condensent d'éphémères noyaux sémantiques, toujours lacunaires, mais qu'on peut cependant indexer sur deux isotopies maîtresses. L'une d'elles, dont il a déjà été question, articule le texte autour d'un contraste chromatique entre /noir/ et /blanc/, associés soit orthosémémiquement, soit et le plus souvent rhétoriquement (par métonymies interposées), de manière à multiplier antithèses et oxymores : «l'ombre faible - écume à l'ombre - de chair »; «j' - étais cet astr - $e$ je suis ce noi - $r$ que tu préfe - res»; «cygne blanche - parole enfouie - sous la masse $n$-octurne »; «le rire ce sou - pçon d'ébène l - es lilas tu dor - s», ou encore «belle un soir - blanche». J'ai suggéré plus haut que ces associations noir/blanc pouvaient être mises en concordance avec le mode d'inscription du texte (gras sur blanc), comme si, à l'inverse de ce qui prévaut d'ordinaire, c'était ici le contenu du poème qui répliquait sa forme extérieure. A moins que ce ne soit le rapport de subordination entre intériorité rhétorique et extériorité typographique lui-même qui soit du coup rendu parfaitement indécidable.

Quoi qu'il en soit, le jeu des couleurs - où interviennent aussi, autres opposées, la bleue et la rouge 43 — conduit à un autre niveau de compréhension, plus diffus mais aussi plus

42 Le texte s'achève en effet sur la mention «FIN». Entre son titre et son mot de conclusion, LXTROHP est ainsi placé entre la marque même de toute Origine textuelle (l'alphabet) et la marque explicite de la Fin. A la lumière de l'analyse qui va suivre, c'est peut-être cela seulement qu'il raconte : entre la naissance et la mort, il n'y a place, il ne devrait y avoir place, indiscernablement, que pour l'aventure de la lettre et du désir, du sens et des sens. Notons en outre que le mot «FIN" intervient dès la première page, isolé en dernière ligne d'un des blocs (la fin est déjà, en logique circulaire, dans le commencement). 
fondamental. S'adressant à l'œil, à la vue, au regard, il introduit en effet au registre général des /cinq sens/, tour à tour nommés ou évoqués métonymiquement par le biais des éléments, saveurs, fragrances, ondes, contacts, dont ils sont les capteurs subtils. Voici d'abord, de loin le plus fréquent, la vue, «voyageuse de cils par le ch - emin de la co - uleur »; puis l'ouie, tout à sa faculté d' «écoute " malgré «cette oreille trop petite»; arrive aussi le toucher sur la «feuille de chair» avec ses «doigts de neige»; après quoi, le goût, avec son site buccal, ce qui l'alimente — «de quoi de nour - ris -tu main b-ouche double», «j'étais le seul bou - langer » - et sa capacité à discriminer les saveurs «parure de sel », «ni le rat sucre - sucre ni le loup» — ; vient enfin l'odorat, «respiration» percevant la «mise de parfu - ms » dans «l'aigr - ette où je res - pire »44. Mais cette profusion des sens n'est pas seulement abondance et répétition, elle est aussi synesthésie, télescopage des sensations, pouvant agir à trois degrés : soit en corrélant deux ou plusieurs sens : «voyageuse de - cils par le ch - emin de la co - uleur l'aigr - ette où je res pire » (vue + goût 45 + odorat), «j'écoutais ta - respiration» (ouïe + odorat); soit en traduisant un sens par un autre : «de quoi te nour - ris -tu main b-ouche double main parure de - sel et d'ongle» (goût + toucher); soit encore en affectant à un sens les propriétés d'un autre : «murmure l'oe - il» (ouie + vue) ${ }^{46}$. Alliance sensorielle opérée à l'échelle d'un bloc entier dans l'exemple suivant, où interfèrent le goût, la vue et le toucher (marqués en maigres) :

\author{
L'EAU LA GORGE \\ ET LA TRENTIEM \\ E DOUCEUR CER \\ TAINEMENT A G \\ AUCHE QUAND LE \\ MIROIR DORT D \\ ANS SON HABIT \\ DE CHAIR
}

Tous sens mêlés, la sensation s'éprouve au plus intense. Jusqu'à devenir mode euphorique de connaissance immédiate, pur être-

Par exemple : "APRES LA BLEUE-LA ROUGE-LA SEU-LE COULEUR JE L'-AFFIRME-LE JOY-AU DES FLEUVES».

44 Ces citations ne sont que quelques prélèvements sur un tissu très dense.

$45 \mathrm{Si}$ l'on veut bien considérer que la coupure du mot «aigrette» dégage "AIGR(E)», soit l'une des quatre saveurs.

46 Ajoutons, s'agissant de ce dernier exemple, que «murmure», en tant que production vocale, est indexable dans la catégorie du Logos. 
au-monde rejetant les béquilles de la pensée : «la boucle - nourrie de sens - les seins et ce - que je ne pense pas». Façon sans doute, pour Goemans, d'affirmer poétiquement le primat du sensible sur l'intelligible, en un texte qui manie mots et lettres et s'adresse au sens du regard avant de se prêter à la lecture ou aux spéculations de l'analyse. Mais, fait de signes et d'agencements strophiques, LXTROHP est aussi incantation érotique, où le mélange rhétorique des sens traduit ce désir extasié venant au corps, dans l'ébat amoureux («notre cheval - de foudre»), d'étreindre sans perte l'autre corps, avec le velouté de sa peau, ses parfums, ses saveurs, l'éclat de ses yeux dans l'imminence crispée et frissonnante du jouir («o - ui oui l'herbe - frissonnant de fourmis»).

Unifiant les sensations qu'il émet et reçoit, LXTROHP disperse néanmoins ce corps, ne le désigne que par fragments, métaphores ou allusions latérales ${ }^{47}$. Tradition du Blason oblige, dont les surréalistes, Breton en tête, ont repris le flambeau. Mais ce qui valait pour les poètes de la Renaissance reste encore de mise : à dire le corps dans sa totalité sensible, le langage est impuissant. Il lui faut biaiser, transiter et transiger, procéder par prélèvements, inventer des ruses traversières. Puiser aux ressources rhétoriques de la synecdoque et de la métonymie qui, du corps propre, font un réseau épars de figures et de la «blanche parole enfouie» qu'il «murmure», un puzzle jamais complet de mots toujours en porte-à-faux sur leur signifié supposé. Démarche où entre certes un fétichisme du corps morcelé, fêté par zones et régions, mais aussi une jubilation verbale à répéter le jouissible. Fragmenté, le corps multiplie le plaisir à le dire ; dérobé, il aiguise l'envie de le poursuivre, de le ressaisir.

Evitement dispersif auquel Goemans - ou l'amant qui parle en son texte - n'échappe pas, mais du moins en le sachant et en le proclamant : «où trouverais-je - son corps» ? L'écriture est ainsi engagée dans une quête fascinée, celle d'un corps proliférant et prolifique, insituable et insatiable («de quoi te nour - ristu»), doublement dévorant («b - ouche double»), fait de neige et de feu ${ }^{48}$, «march[ant] d'île en île». De là qu'en de nombreuses occurrences, la destinatrice - tantôt présente au discours sous la forme d'un «tu», tantôt absente sous la forme d'un «elle» — soit

47 La place manque ici pour relever en détail les morceaux épars et les modalités rhétoriques de cette fragmentation - yeux, torse, bras, tête, bouche, main, oreille, doigts, boucle, etc. 
plus ou moins directement associée à des notions de fuite, d'errance, d'oubli ou d'identité changeante : «l'aventurière l'oubliée», «y éta - is-tu», «jamais la - même», «la voix - change», «fuyant une ai - le», «parole enfouie», «voyageuse de cils», «tourbillon la - furtive», etc. ${ }^{49}$ En réaction à cette errante évasion, l'énonciateur, qui se dit «chasseur» enchaîné («ch aines je reste») et à peine assuré d'aboutir («nous nous ren contrerons [...] quelq - ue jour»), réclame de sa fugitive, «forte de - [s]es appels», un message, un signal au moins phatique : «écris-moi hi - rondelle», "pour - quoi ne m'as-tu - pas dit allo al - lo ô revoir»50. Et s'il semble par deux fois toucher au but ou parvenir à rassembler le corps fluctuant qu'il pourchasse, ce n'est là qu'un effet de parole ou de rêve : «que dit la voi - ci le rêve », «le son de m - a voix tu es l'u - nique». Inutile de lire ici le récit brisé de quelque déroute sentimentale : c'est le corps aimé, non l'aimée, qui échappe à la préhension du langage, «grand fan tôme presque - apaisé de fai - m» qu'elle échoue à retenir et à unifier. Il ne revient qu'à l'instant de l'étreinte - «tes bras - à mon cou ma $d$ - emeure »-d'arrêter sa course et de lui recomposer une intégrité, qui n'est ni de discours ni de chair, mais faite de sensations enchevêtrées 51

Sous pareil angle, la construction typographique du texte, aussi autonomisée nous a-t-elle paru, laisse entrevoir cependant à quelle sollicitation figurative elle a pu notamment répondre. Si l'emphase des capitales et des caractères gras annonce et puis relance la dimension célébrative du texte, l'agencement dispersif

${ }^{48} C f$. parmi d'autres occurrences du même ordre: "VOS DOIGTS-DE NEIGE ô UN-BAL UN BAL LE F-EU SINON».

$49 \mathrm{Du}$ coup se propose en ultime hypothèse l'idée que le $\mathrm{Z}$ manquant de l'alphabet titulaire y figure l'absente et la voyageuse dont le texte décrit la quête.

50 Jeu métagraphique remarquable puisqu'en substituant «ô revoir» à «au revoir», en vertu de leur homophonie, il insiste sur la fonction d'appel du message et opère en même temps une inversion pragmatique du sens («au revoir» s'énonce en principe au moment d'une séparation, tandis qu' «ô revoir» exprime l'aspiration aux retrouvailles). Ajoutons encore que la collocation entre "allo» et "[au] revoir» articule en un même point du discours deux messages à fonction phatique situés en réalité aux deux moments extrêmes d'une séquence de communication (la prise de contact et la rupture du contact).

51 En termes rhétoriques, l'intégrité ne sera donc recomposée que de façon doublement métonymique : si le corps s'unifie dans et par les sensations qu'il suscite ou éprouve, chacune d'entre elles, ainsi qu'on l'a vu, "glisse» vers les autres, via le système englobant des /cinq sens/, par effet de contiguïté référentielle. 
des pages et, en chaque fragment, l'éclatement morpho-syntaxique des phrases accordent quant à eux sa forme «extérieure» à cette recherche éperdue d'un corps épars, à l'identité fuyante. Au registre des célébrations amoureuses, entre L'Union libre de Breton et l'Esquisse d'un hymne d Marthe Beauvoisin de Nougé, entre l'hommage litanique et la narration poétique «hard», LXTROHP occupe ainsi une place singulière, celle d'un texte hybride, à mi-chemin du mot et de l'image, du sensible et de l'intelligible, où le corps des lettres assume au propre sa métaphore originaire. Réseau de signes tracés sur une «légère - feuille de chair». Lettre errante d'un message amoureux, tatoué en caresse sur la peau de la page.

Pascal DURAND

Université de Liège 